\title{
VLBI OBSERVATIONS OF THE 0957+561 GRAVITATIONAL LENS SYSTEM
}

\author{
M.V. Gorenstein (CfA), R.J. Bonometti (MIT), N.L. Cohen (Cornell), \\ E.E. Falco, I.I. Shapiro, N. Bartel (CfA), A.E.E. Rogers (Haystack), \\ J.M. Marcaide (IAA), T.A. Clark (GSFC)
}

\begin{abstract}
A series of VLBI observations of the gravitational lens system $0957+561$ at $\lambda 13 \mathrm{~cm}$ has yielded the positions of the $A$ and $B$ images, the relative magnification of their largest discernible radio structures, and the time variability of their smallest discernible radio structures. These observations have also allowed upper limits to be placed on the flux density of an expected third image. The positions and relative magnification of the $A$ and $B$ images provide new information with which to constrain models of the lens that forms the images. The detection of variations in the flux densities of the cores of $A$ and $B$ suggests that observations at shorter wavelengths may reveal superluminal motion, which may in turn provide a means to measure the relative time delay.
\end{abstract}

\section{INTRODUCTION}

The $0957+561$ gravitational lens system (Walsh, Carswell, and Weymann 1979) is the first known and best studied example of the gravitational lens effect. VLBI observations can contribute to our understanding of this, and other, lens systems through the determination of the observable properties of the images. For two images of a common source, VLBI observations can determine their (i) relative positions to sub-milliarcsecond accuracy from the difference of their interferometer phase delays; (ii) relative magnification from their partially-resolved time-independent brightness distributions; and (iii) relative time delay, $\Delta \tau_{B A}$, from correlations in the evolution of their partially-resolved time-variable brightness distributions. These results can check the basic lensing hypothesis, add new information with which to constrain lens models, and, perhaps, provide a new means with which to estimate the Hubble constant.

In 1981, at $\lambda 13 \mathrm{~cm}$ (Cohen 1985), and in 1983, at $\lambda 13 \mathrm{~cm}, \lambda 6 \mathrm{~cm}$, and $\lambda 3.6 \mathrm{~cm}$ (Bonometti 1985), we observed with VLBI the A and B gravitational images of the quasar Q0957+561. Here, we discuss only the $\lambda 13 \mathrm{~cm}$ data taken at these two epochs. We employed the Mark-III VLBI data acquisition system (Rogers et al. 1983), recording a 56-MHz band (Mode-A), at participating stations located at Effelsberg, FGR (100-m diameter antenna); Onsala, Sweden (25-m); Madrid, Spain (64-m); Green Bank, WV (43-m); Owens Valley, CA (40-m); and Goldstone, CA (64-m). The minimum fringe spacing, obtained on the Effelsberg-Goldstone baseline, was 3.5 milliarcseconds (mas).

In §II we describe models for the brightness distributions of the $\mathrm{A}$ and $\mathrm{B}$ images obtained from our VLBI data, and the properties of the images that follow from these models. In §III we discuss the detection of the radio core of the main lensing galaxy G1 in the $0957+561$ system and the limits on the flux density of a third image of Q0957+561. The following paper in the volume (Falco, Gorenstein, and Shapiro 1987a) presents a model of the mass distribution of the lens constrained, in part, by the data presented here.

201

M. J. Reid and J. M. Moran (eds.), The Impact of VLBI on Astrophysics and Geophysics, 201-206.

(C) 1988 by the IAU. 


\section{OBSERVABLE PROPERTIES OF THE A AND B IMAGES}

In §2.1, we present models of the brightness distributions of $A$ and $B$ and demonstrate that they are images of a common source and have opposite parity, as predicted from models for the lens' mass distribution. In $\S 2.2$, we give our result for the relative position of the images. In $\S 2.3$, we discuss evidence for the time variability of the cores' flux density.

\subsection{VLBI Structure of the Images - Confirmation of the Lensing Hypothesis}

We present in Figure 1 a model ("Model I") for the brightness distribution of each image obtained from the 1981 data (Cohen 1985; Cohen et al. 1987). We modeled each image with four elliptical Gaussian components: two compact components (Core and Jet 1) and two extended components (Jet 2 and Jet 3 ). The model parameters were constrained by correlated flux densities, closure phases, and difference phases (for definitions of difference phases, see $\S 2.2$ ). The $\chi^{2}$ contribution per degree of freedom (DOF) for the (combined) models is 2.2. For these preliminary results, the errors in the magnification parameters and in the $\mathrm{A}-\mathrm{B}$ difference position presented in Table 1 , and in the data presented in Figure 2, are all a factor of two larger than the statistical errors. Final values for these errors will be presented in Cohen et al. (1987).

The approximate correspondence in relative position, flux density, size, shape, and orientation of the respective components in $\mathrm{A}$ and $\mathrm{B}$ suggests that they are images of the same source. We test these correspondences quantitatively as follows: First, we note that the angular extent of each image ( $\sim 60$ mas) is small compared to the arcsecond extent of the lens, suggesting that over the image's brightness distribution, the deflection caused by the lens changes linearly with position. Geometric optics implies then that the brightness distribution of an image should be related to the source's distribution by a three-parameter transformation that corresponds to a magnification and an astigmatism. Further, the brightness distribution of one image can be related to that of the other image by a four-parameter transformation, described by the four elements of a $2 \times 2$ "magnification matrix" [see Gorenstein et al. (1984) for details]. This magnification matrix can be estimated from corresponding components of images observed at one epoch, provided the time evolution of those components on the time scale of the relative time delay can be neglected.

For the 1981 data, we also used a second model ("Model II") that had parameters for the structures of the images and for the magnification matrix. The magnification parameters were used to relate only the flux densities, shapes, and orientations of Jet 2 and Jet 3 in the A and B images, and their positions relative to the centers of the respective Core components. As Jet 2 and Jet 3 are at least a few hundred light-years in extent, we concluded that we could neglect any evolution in their structure.

For Model II, the parameters describing the flux densities, shapes, and relative positions of the Core and Jet 1 components were estimated independently for both images, since, because of possible evolution, these parameters may not be related accurately by the magnification matrix. As these components are only some tens of light years in extent, or less, their brightness distribution could evolve significantly over a time delay of order $1 \mathrm{yr}$.

Inspection of Figure 1 suggests that the B image, aside from parts affected by evolution, can be obtained from the $\mathrm{A}$ image by a magnification of about 1.2 applied along the jet axis. Model I shows that the ratio of flux densities of Jet 2 and Jet 3 is $B / A \sim 0.6$. This result suggests a demagnification along an axis perpendicular to the jet axis of about $0.6 / 1.2=0.5$. Estimates of the magnification parameters conform to these results, as shown in Table 1. The total relative magnification, $B / A$, is $0.64 \pm 0.05$. The $\chi^{2}$ per DOF for Model II is $2.2 ; \chi^{2}$ for Model II is 7 larger than for Model I for an increase of 8 degrees of freedom, which shows that the models are statistically indistinguishable. The estimated values for these parameters are independent of possible systematic errors in the overall calibration, as the magnification depends only on the ratios of flux densities. However, we have not yet checked the sensitivity of the magnification parameters 
to systematic calibration errors that may be produced at each station.

Models for the lens in the $0957+561$ system predict that the A and B images have opposite parity (Young et al. 1981). Model I, presented in Figure 1, confirms this prediction, albeit only at the lowest, least significant, contour level: for example, Jet 2 appears to the left of Jet 3 in A, but to the right of Jet 3 in $B$. To test the significance, we analyzed a third model in which the relative parity of the images was constrained to be positive; $\chi^{2}$ for this third model was larger than for Model I, but by an amount too small to rule out the alternative hypothesis with high confidence.

The apparent existence of the same number of components in each image, their satisfaction of the magnification relationship, including parity reversal, all are consistent with, and therefore serve to confirm, the lens hypothesis.

\subsection{Difference Position}

Because of their close $\sim 6^{\prime \prime}$ separation, both images were observed simultaneously by all antennas during the observations. For each 13 min 'scan', we obtained, for each interferometer, a phase delay for each of the images; we employed the difference of these phases in our astrometric analysis. The difference of these phases eliminates common contributions to both that arise from the atmosphere, instrumentation, and clock behaviors; the difference phase equals the sum of the difference phases due to the images' different sky locations and brightness structures.

We employed these difference phases, together with the visibility amplitudes and closure phases for each source, to obtain a difference position for the Core components in A and B (Cohen et al. 1987), presented in Table 1.

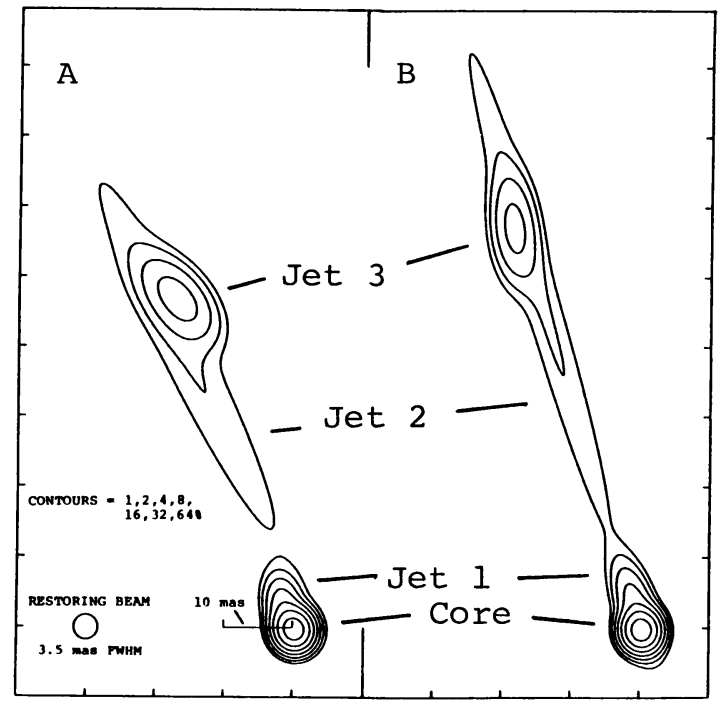

Figure $1-$ The brightness distributions of $0957+561 \mathrm{~A}$ and B. Four elliptical Gaussian components account for the data obtained from the $1981 \lambda 13 \mathrm{~cm}$ VLBI observations for $A$ and for B (Model I-see text). The correspondences in the number and in the properties of the components of the respective models, and the evidence for a change in parity from one image to the other, all support the hypothesis that $A$ and $B$ are images of a single object. 


$$
\begin{aligned}
& \text { Redshifts } \\
& \begin{array}{cc}
\multicolumn{2}{c}{\text { Images }^{a}} \\
1.4136 \quad \pm 0.0002 & 0.36 \quad \mathrm{G1}^{b} \\
\hline 0.01
\end{array}
\end{aligned}
$$

Image and Lens Positions

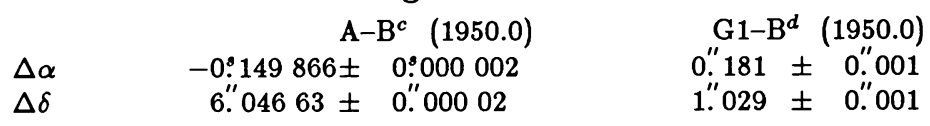

\begin{tabular}{ccc}
\multicolumn{2}{c}{ Relative Magnification Matrix } & (B/A) \\
& Linear Magnification & Direction of Magnification Axis \\
(Eigenvalues) & (Pos. Ang. of Eigenvectors) \\
+1.21 & \pm 0.01 & $+18^{\circ} 9 \quad \pm 0.2$ \\
-0.53 & \pm 0.04 & $-70^{\circ} \quad \pm 10^{\circ}$
\end{tabular}

Relative Magnification

(Product of Eigenvalues) $-0.64 \pm 0.05$

\footnotetext{
${ }^{a}$ From optical observations, Weymann et al. (1979).

${ }^{b}$ From optical observations, Young et al. (1981).

${ }^{c}$ From $1981 \lambda 13 \mathrm{~cm}$ VLBI observations, Cohen et al. (1987).

${ }^{d}$ From $1981 \lambda 13 \mathrm{~cm}$ VLBI observations, Gorenstein et al. (1983).
}

Table 1 - The observable properties of the images and of the lens of the $0957+561$ system. We employ these properties to constrain a model for the mass distribution of the 0957+561 system (Falco, Gorenstein, and Shapiro 1987a).

\subsection{Time Variability of Images.}

The relative time delay, $\Delta \tau_{B A}$, for the images results from the differing lengths and gravitational potentials associated with the respective ray paths. A measurement of $\Delta \tau_{B A}$ is crucial because, first, a detection of definitive correlated variations between the images would provide a final proof of the reality of the gravitational lens effect, and, second, the value for $\Delta \tau_{B A}$ is inversely proportional to the Hubble constant (Falco, Gorenstein, and Shapiro [1985] and references therein). The value for $\Delta \tau_{B A}$, together with a model for the mass distribution of the lens, can yield a value for $H_{0}$ and for the mass of the lens.

In 1983 we re-observed the $0957+561$ system at $\lambda 13 \mathrm{~cm}$ to search for possible time evolution of the Core and Jet 1 in each image. Such evolution, if detected, could lead to a measurement of $\Delta \tau_{B A}$. The models for the Jet 2 and Jet 3 components in the $\mathrm{A}$ and $\mathrm{B}$ images obtained from the 1983 data were consistent with the corresponding models obtained from the 1981 data. However, the flux densities of the Core and Jet 1 in both images changed. In Figure 2, we plot the sum of the Core+Jet 1 flux densities for A and for B for both epochs. (We consider only the sum of these flux densities because the components were barely resolved with the 3 mas resolution of the longest-baseline available, causing the estimates of the individual flux densities to be highly correlated for each image for each epoch.) The Core+Jet 1 flux densities in 1983 can be seen to be significantly lower than in 1981 for both images. Further, the difference between the (scaled) flux densities for $\mathrm{A}$ and $\mathrm{B}$ obtained at the same epoch gives evidence for the time evolution of the flux density of the Core+Jet 1 for the source on the time scale of the time delay: If there were no time 
evolution, the Core+Jet 1 flux density for B, when scaled for the magnification as determined by the outer jets, would equal, within the measurement errors, the Core+Jet 1 flux density for $A$.

A reliable value for $\Delta \tau_{B A}$ cannot be extracted from thesc data; however, we can obtain a model-dependent value. For example, if we assume the decay to be linear in time, we obtain $\Delta \tau_{B A}=4 \pm 1 \mathrm{yr}$ (A varies first); in Figure 2, the open triangles represent the B data translated by this amount, with the corresponding light curve being indicated by the dashed line. The data do not distinguish this light curve from other possible light curves. Although our data do demonstrate that the flux density of the source varies on a time scale comparable to $\Delta \tau_{B A}$, they do not provide a definitive demonstration of correlated evolution of the images, nor do they provide a reliable estimate for the time delay.

Motion of multiple components in a radio source can provide another means with which to determine $\Delta \tau_{B A}$, as pointed out by Vanderriest (1982) and Gorenstein et al. (1984). For example, a core-jet separation could be determined for each image at each of two epochs; then, if the deduced separation velocities were constant, but non-zero and detectable, we could determine for each image the epoch of zero apparent separation. The difference in these epochs yields $\Delta \tau_{B A}$. Our original goal in performing a second epoch observation at $\lambda 13 \mathrm{~cm}$ was to detect a change in the separation of Jet 1 from the Core in each image. Our 1983 data showed that these structures were still barely resolved, and no statistically significant change in their separation from 1981 to 1983 was evident.

The changes in the flux density of the images over two years demonstrates that the brightness distribution of the source is evolving on a useful time scale. This evolution gives us hope that similar evolution may occur at shorter wavelengths, where we may be able to detect observable changes in the images' brightness distributions. We are planning observations at $\lambda 6 \mathrm{~cm}$ and, ultimately, at $\lambda 3.6 \mathrm{~cm}$, in order to search for evidence for such structural evolution.

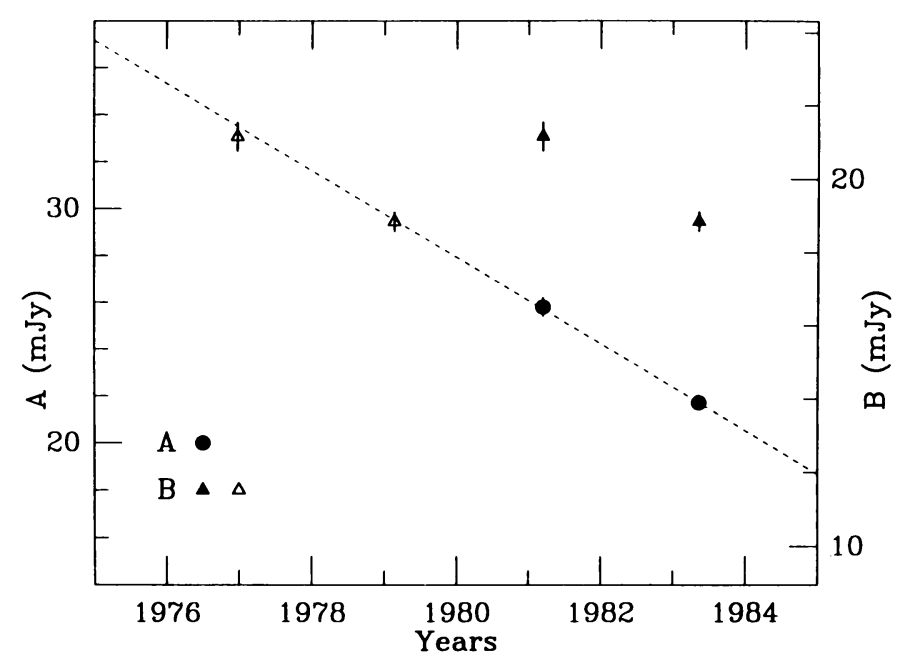

Figure 2 - The flux densities of the Core+Jet 1 components in $A$ and in $B$ at $\lambda 13 \mathrm{~cm}$ in 1981 and 1983. The $A(B)$ flux densities are plotted with respect to the left- (right-) hand vertical axes; the scale of the latter axis reflects the ratio of the image magnification $(B / A=0.64)$. These data are consistent with $\Delta \tau_{B A}=4 \pm 1$ years, if the flux density of the Core+Jet 1 is assumed to decay linearly (see text). 


\section{WHITHER THE THIRD IMAGE?}

Theories of lensing predict that every transparent lens should produce an odd number of images, except for "singular" relative orientations of source, lens, and observer (Burke 1981). Our search for a third image in the $0957+561$ system using our 1981 data led to the discovery of a weak $(0.6 \pm 0.1 \mathrm{mJy})$, compact $(<2 \mathrm{mas})$ radio source, $\mathrm{G}^{\prime}$, located on the sky at or near the center of the foreground galaxy G1 (Gorenstein et al. 1983). The size, flux density, and location of $\mathrm{G}^{\prime}$ were consistent with it being either a third image of Q0957+561, radiation from G1, or, perhaps, some combination of the two.

Our 1983 data revealed that the correlated flux density of $\mathrm{G}^{\prime}$ increased from its 1981 value by nearly $100 \%$ to $1.0 \pm 0.1 \mathrm{mJy}$. If $\mathrm{G}^{\prime}$ were an image, then this increase could be due to a corresponding brightening of the source, or microlensing by a compact mass in G1 traversing the ray path. However, the flux density for the nearby $B$ image decreased by about $5 \%$ during this period, while our models for the lens predict that the propagation times for $B$ and the possible third image $G^{\prime}$ should differ by, at most, a few weeks (Falco, Gorenstein, and Shapiro 1987b). Further, the apparent angular size of such a demagnified image should be no less than $\sim 0.1$ mas ( $1 \mathrm{ly}$ for $H_{0}=100 \mathrm{~km} \mathrm{sec}^{-1} \mathrm{Mpc}^{-1}$ ); so a suitable microlens must have a speed near or greater than $c$ in order to traverse this distance in $2 \mathrm{yr}$. A straightforward interpretation of these results implies that $G^{\prime}$ is not the third image, but rather the radio core of $G 1$. The variations in, and the values of, the flux density of $\mathrm{G}^{\prime}$ are within the range expected from observations of the cores of other elliptical galaxies (e.g., Sramek 1975).

We combined the most sensitive data from both the 1981 and 1983 observations and searched for evidence for an additional image (" $\left.\mathrm{C}^{\prime}\right)$ over a field of width 1.0 in $\alpha$ and 1.6 in $\delta$ that was centered $1^{\prime \prime}$ due north of $B$. Our search yielded no evidence for $C$, setting a $(5 \sigma)$ limit of $0.2 \mathrm{mJy}$, or one part in 80 of the flux density of the compact Core in the $\mathrm{B}$ image. A magnification ratio $(C / B)$ of less than one part in 80 , implies, according to one simple mass model for the G1 lens galaxy, that $\Delta \tau_{C B}$ is less than 3 weeks, the separation of $C$ from the center of G1 is less than 0.2 , and the surface mass density at the center of $\mathrm{G} 1$ is greater than eight times the critical surface mass density (Falco, Gorenstein, and Shapiro 1987b). The critical surface mass density for a given lens system is determined only by the redshifts of the lens and the source and by the cosmology (see Falco, Gorenstein, and Shapiro 1985). For the $0957+561$ system and for $H_{0}=100 \mathrm{hm} \mathrm{sec}^{-1} \mathrm{Mpc}^{-1}$ and $q_{0}=0$, the critical surface mass density is $4.4 \times 10^{10} h^{-1} \mathrm{M}_{\odot} \operatorname{arcsec}^{-2}$.

We gratefully acknowledge support from NSF grants AST8519763 and PHY8409671. One of us (RJB) acknowledges fellowship support from the Hertz Foundation.

Bonometti, R. J. 1985, Thesis, Mass. Inst. of Tech.

Burke, W. L. 1981, Ap. J. (Lett.), 244, L1.

Cohen, N. L. 1985, Thesis, Cornell University.

Cohen, N. L., Gorenstein, M. V., Shapiro, I. I., Rogers, A. E. E., Bonometti, R. J., Falco, E. E., Bartel, N., and Marcaide, J. M., 1987, in preparation.

Falco, E. E., Gorenstein, M. V., and Shapiro, I. I. 1985, Ap. J. (Lett.), 289, L1.

Falco, E. E., Gorenstein, M. V., and Shapiro, I. I. 1987a, in this volume.

Falco, E. E., Gorenstein, M. V., and Shapiro, I. I. 1987b, in preparation.

Gorenstein, M. V. et al. 1983, Science, 219, 54.

Gorenstein, M. V. et al. 1984, Ap. J., 287, 538.

Rogers et al. 1983 Science, 219, 51.

Sramek, R. 1975 Ap. J. (Lett.), 198, L13.

Vanderriest, C. 1982, Astron. Astrop., 106, L1.

Walsh, D., Carswell, R. F., and Weymann, R. J. 1979, Nature, 279, 381.

Weymann, R. J. et al. 1979, Nature, 285, 641.

Young, P. et al. 1981, Ap. J., 244, 736. 